\title{
Feasible Solutions for Precise 3D Printing: The Simulation and Proposal
}

\author{
Jingxuan Lyu ${ }^{1, a, *}$, Hui Shen ${ }^{2}$, Rui Wang ${ }^{2, b}$, Natalie Rudolph ${ }^{3, c}$ \\ ${ }^{1}$ Department of Mechanical Engineering, University of Michigan, Ann Arbor, MI, USA \\ ${ }^{2}$ Instrumentation Science and Opto-electronics Engineering, Beihang University, Beijing, China \\ ${ }^{3}$ Department of Mechanical Engineering, University of Wisconsin Madison, WI, USA \\ ajxlyu@umich.edu, ${ }^{\mathrm{b}}$ wangr@buaa.edu.cn, ${ }^{\mathrm{c}}$ natalie.rudolph@wisc.edu
}

Keywords: 3D Printing, Control System Modelling, Simulation, Adaptive Control

\begin{abstract}
D printing, a kind of emerging technology in additive manufacturing (AM), has been applied in many areas and aroused significant interests in academia and industry, for it promotes the development of democratizing design and manufacturing at lower overall costs. However, most 3D printers use open-loop stepper motor in their extrusion systems, which leads to unstable printing quality because it cannot change extrusion speed accordingly when manufacturing defects occur. Addressing this problem, this paper focuses on improving 3D printer feeding motor's ability of responding to inconstant filament diameter and provides a feasible solution including a conceptual design for feeding motor adaptive control as well as the possible printer configurations. In addition, by comparing series simulation results between the performance of stepper motor and servomotor operating within 3D printer feeding system, we conclude that servomotor can achieve and stabilize at a target value in a shorter time, thus inconstant extrusion caused by filament diameter variations and the slippage can be solved.
\end{abstract}

\section{Introduction}

Additive manufacturing (AM), which builds up 3D objects by adding layer-upon-layer of material, has been applied successfully into commercial use. Among different AM technologies, 3D printing, which uses the principle of Fused Deposition Modelling (FDM), is the most popular technique because it is relatively cheap and easy to set up. In FDM, a print head melts and deposits a thread of thermoplastic material onto a substrate. After being extruded through the print head, the material solidifies and attaches to the previous layers, and thus the building process begins [1]. By providing more design flexibility, reducing the need for assemblage, and improving time and cost efficiency in production run, 3D printing extends the possibilities of the modern production system and has received a lot of popular press attention [2]. It is also predicted that it could be used as a consumer technology business model and even enhance student engagement in education [3], [4].

Although $3 \mathrm{D}$ printing is a valuable addition to classic manufacturing, it lacks adequate monitor and control system to achieve its full potential on parts' mechanical and surface qualities. Currently, several research has been conducted to monitor extrusion process and to improve surface quality. Augmented reality technique is used to monitor the whole printing process by detecting possible printing errors when geometry mismatching happens [5]. A segmentation method is proposed to improve surface quality, which generates parts with higher haptic and visual quality by printing partitioned 3D model and then assembling it following specific order [6]. It is also possible to detect the material deposition status by measuring the current supplied into the motor since the load on the feed motor is related to the extrusion pressure on the nozzle [7]. Furthermore, real-time filament slippage is measured and reduced by implementing closed-loop control on printing extruder using difference between feeding gear speed and filament speed as feedback, along with microscope video camera and image processing [8].

Unfortunately, since the extrusion speed is often set ahead, most research assumes that there is constant extrusion throughout the printer extruder, which is not true. Defects in the solid filament such as deviations of diameter and voids are unavoidable and unpredictable. Feeding filament with 
defects at a constant feed rate would result in inconsistent extrusion, which in turn lowers the quality of the printed part. Our research objective is to improve FDM 3D printer feeding motor's ability of responding to inconstant filament diameter and provides a feasible solution including a conceptual design for feeding motor adaptive control.

\section{Feeding System of 3D Printer}

Main components in feeding system of 3D printer are print-head and feeding motor. The printhead consists with contact pressure wheel, grooved feeding gear, stepper motor, heater, and nozzle, as shown in Figure 1 (a) and (b) [9]. When extrusion begins, the grooved feeding gear, driven by a pinion on the stepper motor, grasps the solid filament and send it into the print-head. A contact pressure wheel applies pressure to the solid filament as a help for the feeding process. After the solid material is fed into the print head, the heated nozzle would heat the solid filament to melt, which is shown in Figure 1(b). Then the polymer melt is extruded through the die at the tip of the nozzle. A 3D object is then built by extruding beads in a layer-by-layer manner.

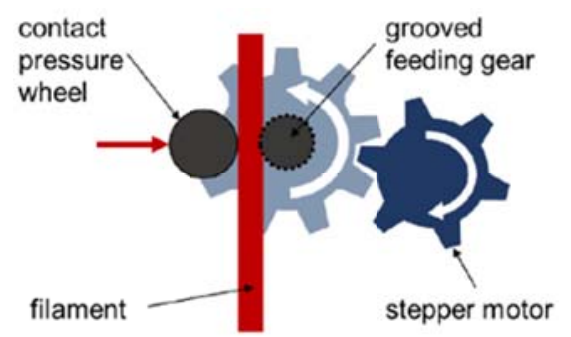

(a) Components of extruder

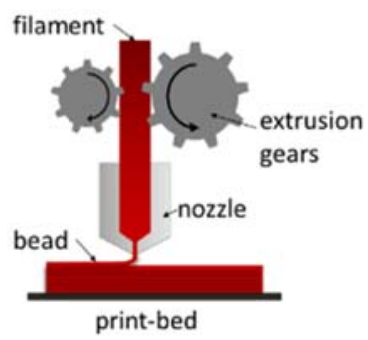

(b)

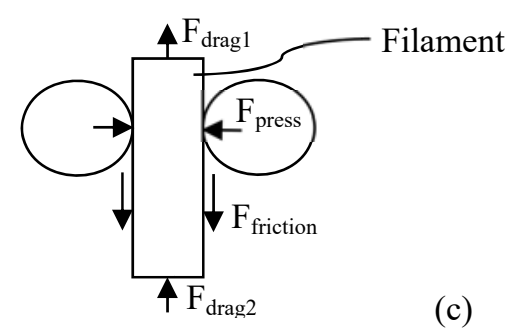

(c) Force analysis

Figure 1 Components in feeding system and force analysis at filament feeding region.

\subsection{Motor Load Analysis}

To choose and simulate an appropriate type of motor for 3D printer feeding system, it is necessary to estimate the load torque and load moment. Force analysis is performed on the filament feeding region to compute for the load torque, which is represented as Figure 1(c). To feed filament into the nozzle, driving force is required to be larger than the sum of drag force and friction. Total pressure within the nozzle can be derived based on the geometry of nozzle by Eq. 1, where $1_{1}$ is length of straight region of nozzle, $l_{2}$ is length of tapered region of nozzle, $1_{3}$ is length of the die, $d_{1}$ is diameter at straight region, $\mathrm{d}_{2}$ is diameter at die, $\mathrm{Q}$ is volumetric flow rate within the nozzle, $K_{d}$ is the ratio between $\mathrm{d}_{1}$ and $\mathrm{d}_{2}, \mathrm{n}$ is pressure gradient and flow rate coefficient, $K_{p}=-\pi_{p} u_{0} r_{0}\left(\frac{4}{\pi r_{0}}\right)^{n}$, $\pi_{p}$ is pressure gradient, $\mathrm{u}_{0}$ is viscosity, and $g\left(K_{d}\right)=\frac{K_{d}\left(K_{d}^{3 n}-1\right)}{K_{d}-1}$.

$$
\Delta P=\frac{K_{p} Q^{n}}{d_{1}^{3 n+1}}\left[l_{1}+\frac{g\left(K_{d}\right)}{3 n} l_{2}+K_{d}^{3 n+1} l_{3}\right]
$$

\subsection{Relationships Among Key Parameters and Feed Rate}

Since filament feed rate depends on motor speed and filament diameter in turn affects motor speed, it is important to know the ideal motor feed rate. To calculate the ideal feed rate, deriving relationships among printing parameters and feed rate is a prerequisite, which is based on following assumptions:

i. Filament is of perfect roundness and has constant density during printing process;

ii. The nozzle is unclogged and the only defect of filament is variation of diameter, meaning that there is no void within the filament.

From these assumptions, it follows that input volumetric flow rate is equal to output volumetric flow rate. Eq. 2 demonstrates that the volumetric flow rate into the print-head is determined by feed rate $v_{\text {feed }}$ and cross sectional area of the filament $A_{\text {filament }}$, and that volumetric flow rate out of the 
nozzle is further related to solidity ratio $\mathrm{SR}$, bead width $\mathrm{w}_{\text {bead }}$, layer height $\mathrm{h}_{\text {layer }}$, and print speed $\mathrm{V}_{\text {print. }}$

$$
\dot{V}_{\text {input }}=v_{\text {feed }} \times A_{\text {filament }}=S R \times w_{\text {bead }} \times h_{\text {layer }} \times v_{\text {print }}=\dot{V}_{\text {output }}
$$

\section{Motor Simulation}

Considering essential elements for motor simulation, such as motor volume, torque output, and operating power, hybrid synchronous stepper motor and permanent-magnet synchronous servomotor are simulated based on their mechanisms and their simulation results are compared.

Key feature to describe hybrid synchronous stepper motor is electromagnetic torque $T_{e}$, which is generated by the interaction among phase current, flux, and detent torque $T_{d m}$. Those three parameters mentioned are further related to number of teeth $\mathrm{p}$, maximum flux $\psi_{m}$, and position angle $\theta$, as shown in Eq.3. Moreover, electromagnetic torque $T_{e}$ can also be expressed as Eq.4 in a dynamic manner, where $J$ is total moment of inertia, B is viscous damping coefficient, $\omega$ is angular velocity, and $\mathrm{T}_{\mathrm{L}}$ is load torque [10].

$$
\begin{array}{r}
T_{e}=-p \psi_{m} \sin (p \theta)-p \psi_{m} \sin \left(p \theta-\frac{\pi}{2}\right)-T_{d m} \sin (2 p \theta) \\
T_{e}=J \frac{d \omega}{d t}+B \omega+T_{L}
\end{array}
$$

As for permanent-magnet synchronous servomotor, its mathematical model is built on following assumptions:

i. Coils are symmetrically distributed, wear on the iron core is negligible, and the generated magnetomotive force along air gap follows sinusoidal distribution;

ii. There is no saturation of magnetic circuit, mutual inductance and self-inductance of each coil are constant, and effect of frequency and temperature change on winding resistance is insignificant.

Final mathematical model of permanent-magnet synchronous servomotor can be described through voltage and flux linkage in Eq.5, as well as electromagnetic torque and dynamic expression in Eq.6. Voltage u, flux linkage $\psi$, current $\mathrm{i}$, and electromagnetic torque $T_{e}$ are first obtained in Cartesian coordinate system, and then they are converted to dq0 coordinate system via Park and Clarke transformations, where subscripts $\mathrm{q}$ and $\mathrm{d}$ stand for axis in $\mathrm{dq} 0$ coordinate, $\mathrm{R}$ is rotor resistance, $T_{L}$ is load torque, $P_{n}$ is number of pole-pairs, $\mathrm{L}$ is inductance, $\psi_{f}$ is permanent magnet flux, $\omega$ is motor angular velocity, $J$ is total moment, and $C$ is damping ratio [11].

$$
\begin{gathered}
\left(\begin{array}{c}
\dot{i_{q}} \\
\dot{\omega}
\end{array}\right)=\left(\begin{array}{cc}
-\frac{R}{L} & \frac{P_{n} \psi_{f}}{L} \\
\frac{1.5 P_{n} \psi_{f}}{J} & -\frac{C}{J}
\end{array}\right)\left(\begin{array}{c}
i_{q} \\
\omega
\end{array}\right)+\left(\begin{array}{c}
\frac{u_{q}}{L} \\
-\frac{T_{L}}{J}
\end{array}\right) \\
T_{e}=\frac{3}{2} P_{n} \psi_{d} i_{q}=J \frac{d \omega}{d t}+C \omega+T_{L}
\end{gathered}
$$

Following Eq.1 and Eq.2, motor load torque and accuracy of speed adjustment can be known. For common FDM 3D printer using filament diameter of $1.75 \mathrm{~mm}$, feed rate is $1 \mathrm{~mm} / \mathrm{s}, \mathrm{u}_{0}$ is 20 $\mathrm{kN} \cdot \mathrm{s} / \mathrm{m}^{2}, \mathrm{n}$ is $1 / 3, \mathrm{~K}_{\mathrm{p}}$ is $1.9855, \mathrm{~K}_{\mathrm{d}}$ is 4 , and feeding gear radius is $5 \mathrm{~mm}$. After plugging parameter values into the equations, driving force $\mathrm{F}_{\text {driving }}$ should be larger than $30 \mathrm{~N}$, and thus load torque on motor should be larger than $0.15 \mathrm{~N} \cdot \mathrm{m}$ after multiplying driving force with feeding gear radius. Furthermore, giving feeding gear diameter is $10 \mathrm{~mm}$, filament diameter is $1.75 \mathrm{~mm} \pm 0.05$, print speed is $30 \mathrm{~mm} / \mathrm{s}$, solidity ratio is 1 , bead width is $0.667 \mathrm{~mm}$, and layer height is $0.4 \mathrm{~mm}$, the motor speed is calculated within the range of $18.45 \mathrm{rpm}$ and $21.65 \mathrm{rpm}$, meaning that the motor should be able to adjust its speed at an accuracy less than $3 \mathrm{rpm}$.

Provided with motor load analysis and feed rate parameters, stepper motor of type SS1703A15A and servomotor of type HF-KP13 made by Mitsubishi Electric are chosen to be simulated. Among seven general modules used in simulations for both types of motors, speed setup, load, and display modules have same parameter settings, respectively: (1) speed setup module assigns two speed 
conditions, one is $0 \mathrm{rpm}$ to $3 \mathrm{rpm}$ for testing general accuracy, and the other one is $2.8 \mathrm{rpm}$ to $3 \mathrm{rpm}$ for testing responding ability; (2) input of load module is set to $0.15 \mathrm{~N} \cdot \mathrm{m}$ from Eq.1; (3) display module allows simulation results to be viewed and analyzed. In terms of feedback controller, microstepper, drive, and motor modules, specific function blocks are used under different simulation conditions, which will be explained in following sections.

\subsection{Stepper Motor Open-loop Simulation}

To simulate stepper motor performance under open-loop control, stepper motor block is used as the motor module. Simulation begins with micro-stepper, which divides motor's basic step to designated step number and sends to drive module after setting motor speed. Then Pulse-width modulation (PWM) technique is used to control the current supplied to motor in the drive module. PWM acts like a switch by comparing the difference between assigned current and real supplied current with a pre-set current value $0.005 \mathrm{~A}$. If the difference is larger than $0.005 \mathrm{~A}$, then it allows power supply to generate higher current to achieve the target value. The block diagram is shown in Figure 2(a), and notice that feedback controller is not used in open-loop simulation.

During the simulation, effect of number of micro-stepping and open-loop motor performance are studied. Motor starts from stationary and after 0.01 second, speed is set to $3 \mathrm{rpm}$, using number of steps of 64 and 512, respectively. The results in Figure 2(b) and (c) show that error decreases and signal becomes more steady when number of step increases. However, regardless of how many steps are used, the steady state value does not stabilize at target value, which may be caused by step loss.

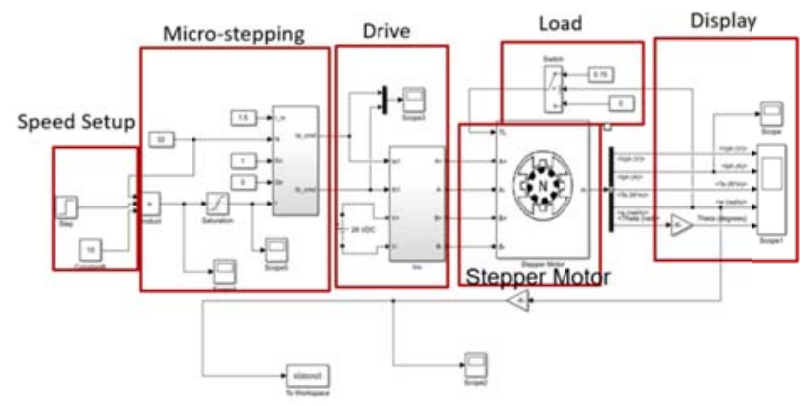

(a) Open-loop simulation block diagram

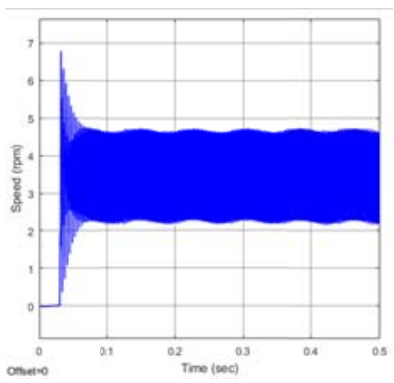

(b) 64 micro-stepping

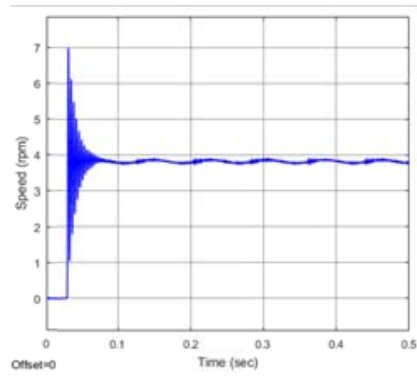

(c) 512 micro-stepping

Figure 2 Stepper motor open-loop simulation block diagram and results.

\subsection{Stepper Motor Closed-loop Simulation}

Based on open-loop stepper motor, PID controller is added to previous model as the feedback module at 512 micro-stepping. PID controller takes the difference between motor speed and target speed as input and outputs micro-stepping frequency to micro-stepping module. Assuming all motor components function well and there is no slippage, there is a linear relationship between motor speed and filament feed rate.

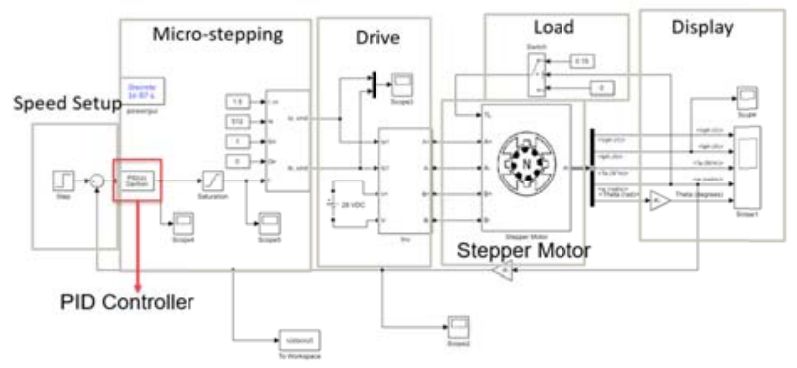

(a) Closed-loop simulation block diagram

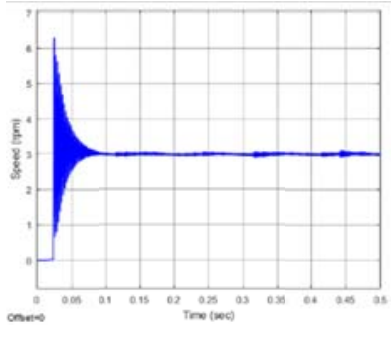

(b) $0 \mathrm{rpm}$ to $3 \mathrm{rpm}$

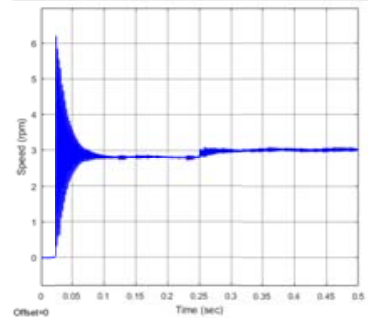

(c) $2.8 \mathrm{rpm}$ to $3 \mathrm{rpm}$

Figure 3 Stepper motor closed-loop simulation block diagram and results.

In addition to previous speed settings, closed-loop stepper motor is also tested with its ability to respond to speed adjustment: motor starts from $2.8 \mathrm{rpm}$ and after 0.25 second the target speed is set 
to $3 \mathrm{rpm}$. The simulation results are demonstrated in Figure 3(b) and 3(c). From simulation results, motor speed achieves the target value in both cases. Therefore, problem of step loss is solved. Time constant in first case is $150 \mathrm{~ms}$, and error is within $0.2 \mathrm{rpm}$; while it only takes $0.08 \mathrm{~ms}$ to reach steady state in second case. If there is no slippage, it is promising that print head can achieve constant extrusion by adding a closed-loop control on the feeding motor.

\subsection{Servo Motor Closed-loop Simulation}

Similar with simulation on stepper motor under closed-loop condition, servomotor is also simulated under same speed settings to compare with performances of closed-loop stepper motor. Permanent magnet synchronous machine is used for motor module. The speed error, calculated as the difference between set speed and measured speed, is the input for the PI controller. In combined with PI controller and vector control, three phase voltage can be obtained and thus drive the motor. Simulation block diagram and results are shown in Figure 4, and notice that micro-stepper is not used for servomotor.

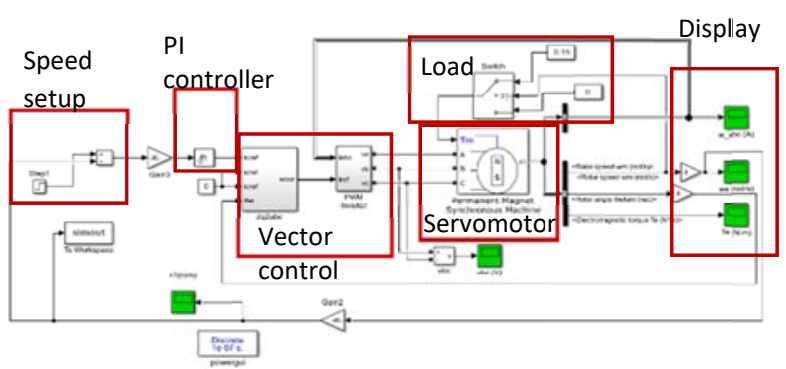

(a) Servomotor simulation

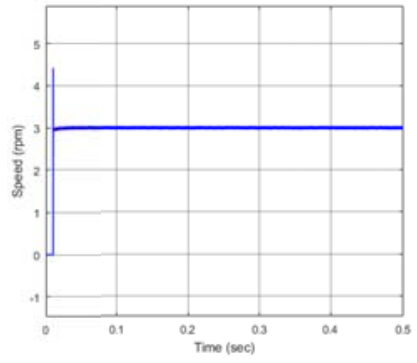

(b) $0 \mathrm{rpm}$ to $3 \mathrm{rpm}$

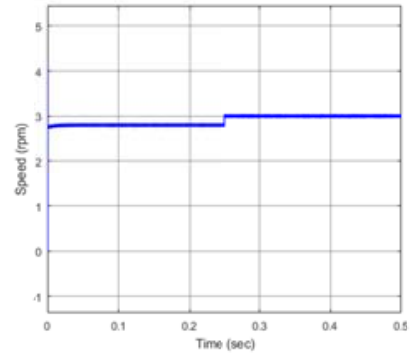

(c) $2.8 \mathrm{rpm}$ to $3 \mathrm{rpm}$

Figure 4 Servomotor closed-loop simulation block diagram and results.

It is observed that it takes $0.15 \mathrm{~ms}$ for servomotor to accelerate from 0 to $3 \mathrm{rpm}$ with an uncertainty of $0.04 \mathrm{rpm}$, and that it takes $0.1 \mathrm{~ms}$ for servomotor to adjust speed from $2.8 \mathrm{rpm}$ to 3 rpm. Therefore, servomotor responds faster and is more stable compared with closed-loop stepper motor.

\section{Feasible Solutions for Precise 3D Printing}

By monitoring the real-time filament diameter and implementing cllosed-loop control on extrusion speed, slippage can be significantly reduced [8]. Here inspired by [8], we propose a whole process to achieve precise $3 \mathrm{D}$ printing focusing on adaptive control of feeding motor and provide a possible printer configuration to measure on-line filament diameter.

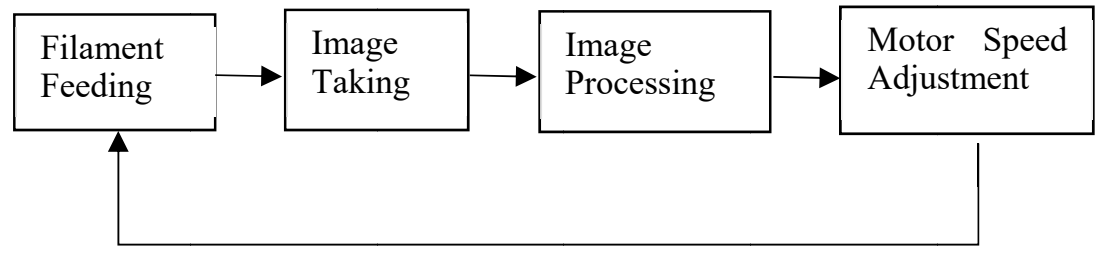

(a) Block diagram of the control process

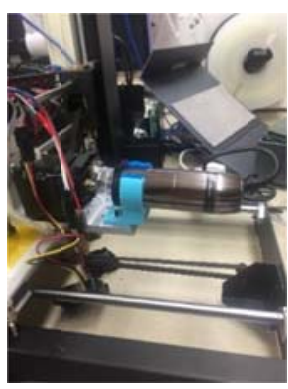

(b) Suggested camera position

Figure 5 Feasible solutions for precise 3D printing.

As shown in Fig.5(a), there are three steps in precise 3D printing: image taking, image processing, and motor speed adjustment. When taking images, device and its position should be considered. For choosing an appropriate device, there are requirements of its resolution, frame rate, and sample rate. As for the device position, microscope camera is suggested to be placed in front of filament feeding region, meaning that camera moves simultaneously with the print head during 
recording (Fig. 5(b)). Given taken images, filament diameter and corners can then be identified by Carsten Steger algorithm, which could achieve subpixel accuracy by extracting lines based on differential geometry and Gaussian smooth [12]. When adjusting motor speed, ideal feed rate is first calculated from Eq.2 and the following control mechanism in [8] can be adopted. In this way, an adaptive control is formed. One thing to notice is that regardless of motor types, it may be hard to change motor speed in a narrow range such as within $3 \mathrm{rpm}$ in this study. Therefore, a gearbox might be required to achieve precise control.

\section{Conclusion}

In this paper, simulation performances of stepper motor under both open-loop and closed-loop situation along with servomotor under closed-loop situation are analysed and compared. Results show that the problem of step loss can be solved after replacing stepper motor with servomotor. And the steady state value agrees to the target value well. Therefore, servomotor has the best performance for improving 3D printer feeding motor's ability of responding to inconstant filament diameter. Finally, a whole process of precise 3D printing using servomotor is proposed, and we will conduct further experiments in the future.

\section{References}

[1] Bikas H., Stavropoulos P., \& Chryssolouris G. (2016) Additive Manufacturing Methods and Modelling Approaches: A Critical Review. The International Journal of Advanced Manufacturing Technology, 83(1-4), 389-405.

[2] Gao W., et al. (2015) The Status, Challenges, and Future of Additive Manufacturing in Engineering. Computer-Aided Design, 69(2015), 65-89.

[3] Brooks G., Kinsley K., and Owens T. (2014) 3D Printing as A Consumer Technology Business Model. International Journal of Management \& Information Systems, 18(4), 271-280.

[4] Rosen D. (2014) Design for Additive Manufacturing: Past, Present, and Future Directions. Journal of Mechanical Design, 136(9), 090301.

[5] Ceruti A., Liverani A., and Bombardi T. (2016) Augmented Vision and Interactive Monitoring in 3D Printing Process. International Journal on Interactive Design and Manufacturing (IJIDeM), 11(2), 385-395.

[6] Wang W. M., Zanni C., and Kobbelt L. (2016) Improved Surface Quality in 3D Printing by Optimizing the Printing Direction. Computer Graphics Forum, 35(2), 59-70.

[7] Chiyen K., et al. (2015) A Study to Detect a Material Deposition Status in Fused Deposition Modeling Technology. 2015 IEEE International Conference on Advanced Intelligent Mechatronics (AIM), 779-783.

[8] Greeff G. P., and Schilling M. (2017) Closed Loop Control of Slippage During Filament Transport in Molten Material Extrusion. Additive Manufacturing, 14, 31-38.

[9] Koch C. (2016) Fused Filament Fabrication (FFF) Optimization: Mechanical Anisotropy and Solidity Effects in Additive Manufacturing. Master's thesis, University of Wisconsin-Madison.

[10] Zhou Y.F. (2014) Simulation of Stepper Motor Control System Based on Simulink. Master's thesis, Southwest Jiaotong University.

[11] Zhou, Y. (2014) Simulation and Comparative Research on Permanent Magnet Synchronous Servomotor Control Strategies, Master thesis, Harbin University of Science and Technology.

[12] Steger, C. (1998) An Unbiased Detector of Curvilinear Structures. IEEE Transactions on Pattern Analysis and Machine Intelligence, 20(2), 113-125. 